\title{
Tests with Blind Programmers Using AWMo: An Accessible Web Modeling Tool
}

\author{
Filipe Del Nero Grillo and Renata Pontin de Mattos Fortes
}

\author{
Computer Science Department, Institute of Mathematics and Computer Sciences at \\ University of São Paulo, São Carlos, Brazil. Av. Trabalhador São-carlense 400 - \\ Centro, P.O.Box 668. 13560-970 - São Carlos/SP, Brazil \\ \{grillo, renata\}@icmc.usp.br \\ http://www.icmc.usp.br
}

\begin{abstract}
With the increase of public utility services being offered by many organizations, such as companies and government institutions, over the Internet and on other electronic medium, the concern related to the access blind and visually impaired citizens is raised. But still, some types of data remain intrinsically unaccessible such as software models, that are generally represented by visually rich diagrams which are inaccessible to screen readers. The goal of this paper is to categorize and evaluate the navigation strategies of blind users using a web application that allows access to UML class diagrams by using a specialized textual language. The investigation was done by analyzing videos with screen capture that were collected as part of a case study conducted with two blind programmers to evaluate the AWMo efficacy in enabling access to visual diagrams for blind and visually impaired software developers. We identified three navigation strategies adopted by the users during the study and the results of the analysis suggest that the navigation in long texts may become a burden on users memories, despite their strategies to improve their speed, non sequential text navigation methods must be further investigated.
\end{abstract}

Keywords: Web Accessibility, Case Study, Navigation, Modeling, UML.

\section{Introduction}

With the increase of public utility services being offered by many organizations, such as companies and government institutions, over the Internet and on other electronic medium, the concern related to the access of the blind and visually impaired is raised. In fact, a variety of laws all around the world has been established to support the rights of all people, including the disabled people such as the Section 508 [1] in United States and e-MAG (Electronic Government Accessibility Model) in Brazil [2].

Although the legislation helps to enforce accessibility to everyone that produce content and provide services on the web, some types of data remain intrinsically dependent on specific senses, such as vision. The software models, for instance,

C. Stephanidis and M. Antona (Eds.): UAHCI/HCII 2014, Part I, LNCS 8513, pp. 104-113, 2014.

(C) Springer International Publishing Switzerland 2014 
are generally represented as visual diagrams, usually composed of geometric forms and connectors associating the geometric forms.

The models have semantics so the people involved with the software process could communicate rationale in a concise way. Most of the times there is also textual content inside these diagrams, but the meaning of the visual notations is so relevant that the understanding of the content is almost impossible when only listening to the textual content of the model. In this context, the AWMo (Accessible Web Modeler) was developed.

AWMo is a prototype Web tool designed to allow users to model software artifacts using a subset of UML class diagrams in two distinct ways. The first one is a graphical view, in which sighted software developers can draw diagrams in the traditional way by dragging elements and connecting them visually. The second view is a textual view, in which software developers with or without visual impairment can create the exact same elements of the diagrams using a textual language developed specially for AWMo.

Based on AWMo, we have conducted user tests with two blind people to evaluate the proposed approach, the tests are briefly described in Section 4 . This paper uses the data collected during the mentioned user tests to analyze the navigation strategies of blind users during their interaction with a web application that allows access to UML class diagrams by using a specialized textual language. The recordings captured both the screen where the user were interacting with AWMo and the webcam where it was able to capture the facial expressions of the user while they worked on a set of five pre established tasks.

The analysis concentrated on the textual language content navigation inside the text editor, but was also extended to the rest of the web application in an attempt to compare the strategies used in hyper documents and simple text. Although there were several other aspects evaluated to reach objectives of the case study itself, this paper focuses solely on the identification and analysis of the navigation strategies adopted by the two blind programmers while interacting for the first time with the textual editor inside a web application they were not previously familiar with.

The remainder of this paper is organized as follows: Section 2 presents work found in the Literature that relates to the work presented in this paper. Section 3 presents the developed prototype tool. Section 4 briefly describes the case study that was conducted to evaluate the AWMo, from where the data used in this paper was collected. Section [5] evaluates the navigation strategies used by the two blind software developers that participated in the case study both in the application interface and inside the tool's textual editor. Finally, Section 6 presents a discussion over the obtained results and concludes the paper with some final remarks.

\section{Related Work}

There are several studies in the Literature that relates to this work from several different aspects. 
As a Web modeling tool, in 3] the authors have developed GEMSJax, a Web version of the Eclipse GEMS software. It was designed to allow remote access via REST API. Similarly there is SLIM 4 which is also a Web modeling tool, but aims at enabling synchronous collaboration on modeling activities. Both these studies present good quality modeling tools but lack accessibility, in other words, for blind and visually impaired users, it would be just as difficult to use the mentioned tools as it would be to use a desktop modeling tool. This is precisely the point that make AWMo different from its peers, AWMo was set out to be accessible from its inception.

AWMo first prototype version was developed to work with UML class diagrams, in this field the work of King et al. (2004) [5] addressed the access of UML software engineering diagrams by blind people. They have developed a tool called TeDUB that allows read access to four of the twelve UML diagrams and their approach was well-received by the participants who have tested it. However, one of the requirements raised by the users was the possibility of editing the diagrams in addition to read it. Without the ability to modify and create new diagrams the access of blind people is only partial, they are able to access and understand the information they seek to convey but are unable to participate in its construction or collaborate in software modeling activities by using TeDUB. With this in mind, AWMo had its scope narrowed to only one of the UML diagrams, the class diagram, but sought out to allow the user to read, modify and create diagrams.

In [6], the authors compare browsing behavior of blind and sighted users. They made use of a proxy and AJAX technology to collect information about the pages that the subjects were browsing. This study had the participation of 10 blind and 10 sighted people and the results indicated that blind users employ coping strategies when facing accessibility problems and find their way to overcome these problems.

The authors of [7] have conducted studies to analyze the interaction of blind and visually impaired users on the Web. They categorized the main problems that the users faced and identified 17 tactics employed to overcome them that were explained in light of coping theory [8]. More than that, they related the tactics employed by the user with technical problems. Similarly, 9] proposed an automatic analysis method of webpage navigability and confuted a survey with 30 real users to evaluate their behavior on online shopping sites. The results indicated that blind users strongly depend on scanning navigation and a landmark-oriented navigation model was proposed based on their findings.

In 10, a set of nine guidelines was developed to assist the development of text interfaces as alternatives to graphical user interfaces (GUI). During the study they conducted an experiment with 39 blind subjects and discovered that the text interface is more usable for the blind. Therefore, the first approach that was explored with AWMo prototype was to allow access to visual models to blind software engineers using a textual language inside a Web application. 
Finally, 11 reports a case study similar to the one conducted by the authors, they used a semi structured interview to know the participants and an observation phase to collect data about blind users navigation.

\section{The AWMo}

The AWMo is a prototype tool developed by the authors in the search of a proposal that would enable blind and visually impaired software developers to work with graphical software models and diagrams. The prototype is restricted to UML class diagrams in order to evaluate the approach.

The key concept behind AWMo approach is to provide two distinct views where the users can work with UML class diagrams: the graphical view, where sighted software developers can draw diagrams in the traditional way (by dragging elements and connecting them visually) and the textual view, where software developers with or without visual impairment can create the exact same diagrams using a textual language developed especially for AWMo.

The main goal of providing two views for the edition of the same model is that both views allow users to accomplish the same tasks and get the same results. By making all the changes made in one view available to the other, AWMo also foster the use of the models as a communication tool for teams of software developers composed of both blind and sighted people, while it does not represent an overhead to sighted users, since they will not necessarily need to learn the textual language in order to use the tool.

AWMo is a Web application developed in JavaServer Faces (JSF) 2.1. The textual language was developed using Eclipse's Xtext project. The Xtext provides a set of tools to allow the definition of grammars using the Extended BackusNaur Form (EBNF) and generates the resources for textual languages such as the parser of the language, validators and code generators. Also, by making available the Ecore metamodel and the EMF classes for the designed language, it allows programatic access to both models and metamodels. The technology behind AWMo is better described in 12 .

Figure 1 shows the interface of the AWMo's textual editor. We can observe that there is a context box on the top showing to the user which diagram is open and allows the user to close it. On the right of the context box is the accessibility toolbar, it allows the user to enable high contrast, control the font size of the application as a whole and also select the language in which the application is presented. On the left side of the main content area there is a menu that is also affected by the context of the application: if there are no open diagrams it will display only the options "Home" and "Help" while if there is an open diagram it will also display the "Textual view" and "Graphical view" options where the user can quickly switch between both textual and graphical editors for the same open diagram. 


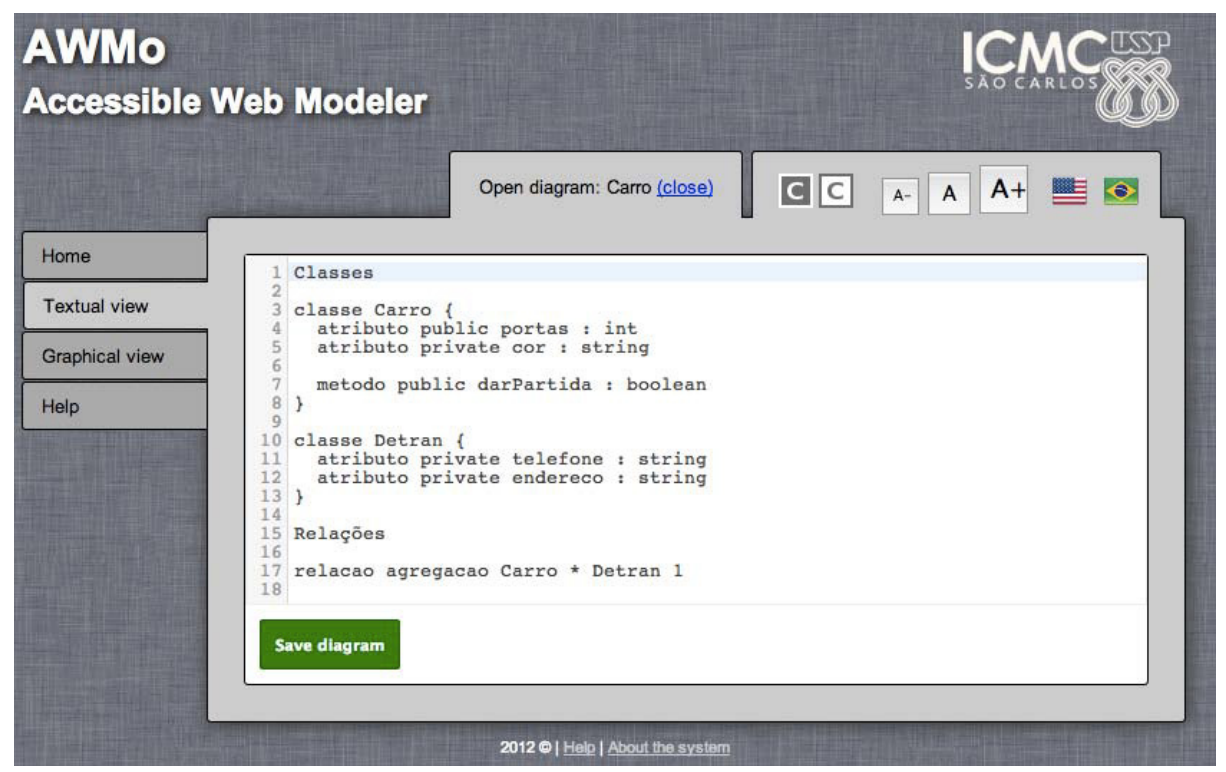

Fig. 1. Screen capture of AWMo interface displaying a diagram open with the textual view

For its interface design, AWMo was developed based on a reference architecture [13, including the visual style, menus and accessibility features such as high contrast, font size control and also the ability to be translated to multiple languages. The fact that both architecture and interface were inherited from the reference architecture and had good accessibility and usability features gave the AWMo tool development a great start up speed. Moreover, it allowed the development be focused on the inner workings and business logic of the proposed modeling environment.

AWMo has earned the second place in Brazilian Web Accessibility Recognition Program - All@Wel11, organized by W3C Brazil, at the Apps and Assistive Technologies category.

\section{Case Study and Data Set}

In order to evaluate the AWMo approach, a case study was conducted with one subject [14] and later on with a second subject that allowed us to gather more data and enrich the work with comparative analysis of the executions.

This study was composed of three phases: an initial interview to know the subjects, an observation phase where the subject performed a series of pre established tasks with AWMo on an instrumented computer and finally a final

${ }^{1}$ http://premio.w3c.br/english/ 
interview to collect the user thoughts and more subjective data regarding the tool and the performed tasks.

Audio of the interviews were recorded and transcribed. The computer screens and webcam were also captured and recorded with the use o Morae Recorder 2 and this data were used to carry out an initial analysis of AWMo approach.

One of the data gathered during the use of Morae was that time each participant took to complete each of the tasks defined for the case study. Figure2 shows the time spent by each participant plotted as lines. We can see that although there were certainly differences in the times spent in each task, the general pattern of the curves is similar. We can see the amount of time increasing in task 3 , reducing around task 4 and spiking again in task 5 . This pattern was expected and relates directly to the level of difficulty of the tasks. The differences in the curves can be related to personal differences from the subjects, one had harder time working with the existing diagrams and the other had a harder time when creating a new diagram from the scratch.

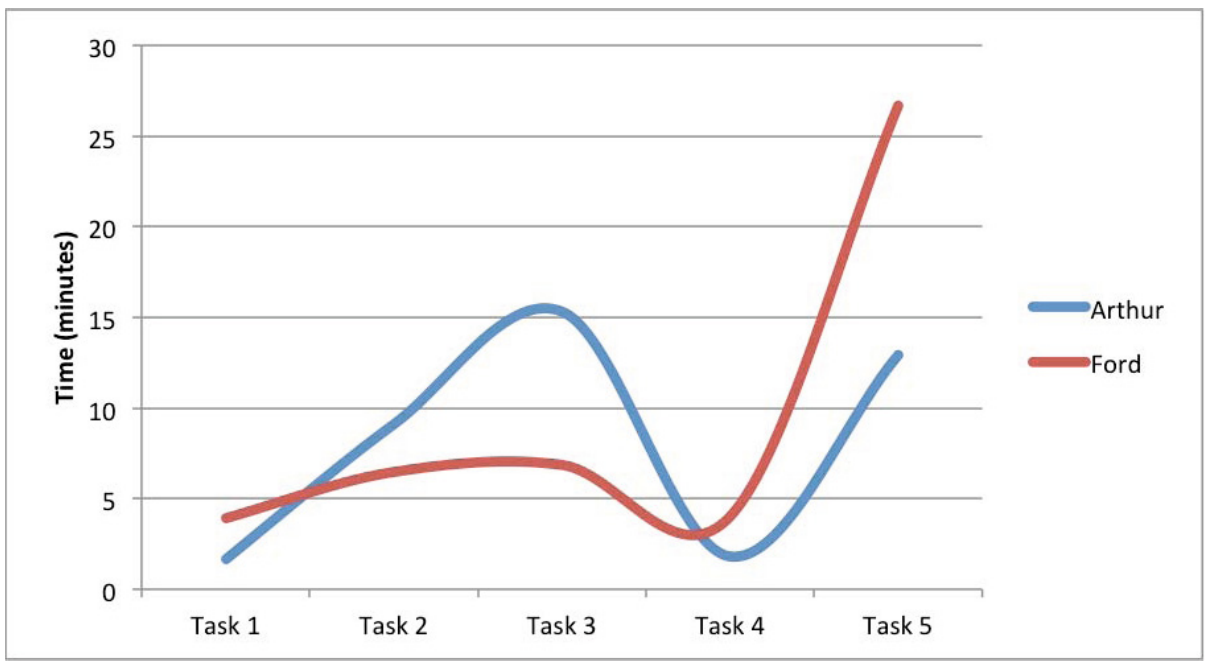

Fig. 2. Chart displaying the time used by the subjects to complete each task proposed by the case study

The results of the case study indicated that the AWMo approach is viable for leveraging collaboration and communication between sighted and visually impaired users on software modeling activities. However, we believe that richness of the gathered data can be further explored and that it can be analyzed from different perspectives. In this study we have analyzed the data evaluating the navigation strategies used by the participants both on the application as a whole and inside textual editor.

\footnotetext{
2 http://www.techsmith.com/morae.html
} 


\section{$5 \quad$ Navigation Strategies}

This work uses the data gathered during the case study mentioned above under a different perspective. By analyzing the footage collected during the case study containing both the computer screen and the users faces we identified navigation strategies the subjects used to navigate inside AWMo and how these strategies evolved during the course of the experiment.

Both participants were completely blind and they made use of the $\mathrm{NVDA}^{3}$ screen reader, the same screen reader they use on their daily use computers.

The task 1 of the case study consisted in the subject using the AWMo accessibility features on the top right toolbar, however since both participants were completely blind and used screen reader to access the web, the features presented were of little help and they both used their time to learn how the interface was structured and the location of the menus, toolbars and other interactive elements. This was the first contact they were having with the tool, all they have read before was a PDF manual teaching them the grammar of the AWMo's textual language.

Since this was their first contact with the web application, both participants adopted scanning techniques, they navigated through the entire page in a moderate pace, understanding the interactive elements and building a mental model of the page organization and the location of its controls. Both participants took less than 2 minutes to perform this task as this is a prototype and, therefore, reduced in number of features. One of the users detected an accessibility problem on the font size controls, they only had the attribute label set and not the title, the label is not read by the screen reader when the user is navigating in virtual mode (using the arrow keys). The other user navigated in forms mode (using tab to move over the focusable elements) and didn't detected this issue.

Task 2 only required the users to read a pre-existing diagram that modeled a simplified version of a banking system and then explain the system to the researcher with their own words. Considering the navigation inside the application interface to open the diagram, the participants navigated considerably faster on the application menus than they did during task 1. One of them used shortcuts of the screen reader to jump directly to the headers $(<\mathrm{h} 1>$ html tags) of the page and consequently getting much faster to the table with the existing diagrams. Considering only the navigation inside the text editor, following the same principle that on Task 1, the participants have scanned the whole text, line by line in a moderate speed because they were having the first contact with the model in question.

For task 3, the participants needed to modify the banking system diagram to specialize the person class into natural person and legal person classes using inheritance between them. In text navigation one of the participants started using a different strategy to access the person class, he jumped to the end of the text and moved upwards to find the desired class. The person class was located much closer to the bottom of the text so it allowed him to get there faster.

3 http://www.nvaccess.org/ 
The same participant also employed the same strategy when navigating the application. After saving the diagram AWMo displays a message informing the user whether the operation was successful or any errors were found in the diagram. For navigating to the message, he navigated backwards, from the bottom of the page until the feedback message. Again the path from below was quicker than the regular top-down approach.

In task 4 participants were expected to remove the savings account class and any of its references from the diagram. Both participants navigated line by line but in a much faster way. One of them only let the NVDA red the first word of the line to identify if it was the line it was looking for and then jumped to the next line. We called it directed scanning, as the user quickly ignored the lines with definition of methods and attributes, since he was looking for a class definition, he listened more carefully the lines with class definitions. The other participant did basically the same with a slight difference: he seemed to have acquired a good notion of the size of each class (in terms of number of lines) and after its declaration (when wasn't the class he was looking for) he jumped line by line so fast that the NVDA wasn't able to not even start reading the sentence. After this short burst, he reduced the speed and listened to the beginning of the next class

Task 5 required the users to create a new diagram using simple specifications provided by the task description. Since the diagram was small and they created it from the beginning, not much navigation strategies were employed inside the text. Except when users were correcting errors pointed by AWMo when they saved the diagram, where line by line navigation sufficed.

\section{Discussion and Concluding Remarks}

Even in situations where there were no common accessibility problems detected, it was possible to observe that the blind users were constantly trying to improve their navigation around the application environment. Three were the strategies adopted by them to speed up their text navigation:

- Bottom-up (web page): sometimes, knowing that the target was closer to the end of the page, the user jumped to the bottom of page and navigated backwards (using shift + tab) to get the point he wanted.

- Bottom-up (text editor): same strategy used on the application, page down key to travel instantly to the bottom of the text area and then move up line by line. If the understanding of the user is correct about the position he is trying to go, this strategy result in less lines read by screen reader and, consequently, less time to reach the objective.

- scanning with landmarks: Fast navigation line by line stoping at landmarks like class definitions. This builds on the knowledge about the estimated number of lines that the class have to speed up the cursor over its lines and get the to desired location even faster.

One interesting fact to observe, is that both participants of the case study rarely used the link to skip to the content of the page. Even though it was there 
and was read by the screen reader, they ignored it and navigated through the menus jumping fast until they reached the page content. According to 9] its is common that most users prefer to scan the whole page and menu to make sure they haven't missed anything.

The possibility of non sequential navigation must be further investigated. We believe that textual navigation will pose as the greatest drawback of the AWMo approach to modeling for blind users. Despite its innovation in allowing edition models by blind and visually impaired users, as systems get complex their models get bigger and bigger and, consequently, strategies for reducing the memory burden on the users might be required.

As a future work, the authors intend to prototype and evaluate different navigation strategies for the textual language of AWMo, allowing the users to navigate throughout the textual content in a non sequential way.

Acknowledgements. We thank all the participants who voluntarily performed the experiments, ICMC-USP for providing the infrastructure that allowed this project to be developed and $\mathrm{CNPq}$ and CAPES for the financial support.

\section{References}

1. US Government: Section 508 of the rehabilitation act (29 u.s.c. $794 \mathrm{~d}$ ), as amended by the workforce investment act of 1998, pp. 105-220 (1998)

2. Executive power: Ordinance $\mathrm{N}^{o} 3$, from 7 of may of 2007. Institutionalizes the Accessibility Model in Electronic Government - e-MAG in scope of the "Sistema de Administração dos Recursos de Informação e Informática" - SISP. Official Gazette of the Union [from] Federarive Republic of Brazil 103 (87), Section 1 (May 2007) ISSN 1677-7042

3. Farwick, M., Agreiter, B., White, J., Forster, S., Lanzanasto, N., Breu, R.: A webbased collaborative metamodeling environment with secure remote model access. In: Benatallah, B., Casati, F., Kappel, G., Rossi, G. (eds.) ICWE 2010. LNCS, vol. 6189, pp. 278-291. Springer, Heidelberg (2010)

4. Thum, C., Schwind, M., Schader, M.: SLIM-A Lightweight Environment for Synchronous Collaborative Modeling. In: Schür, A., Selic, B. (eds.) MODELS 2009. LNCS, vol. 5795, pp. 137-151. Springer, Heidelberg (2009)

5. King, A., Blenkhorn, P., Crombie, D., Dijkstra, S.J., Evans, G., Wood, J.: Presenting UML Software Engineering Diagrams to Blind People. In: Miesenberger, K., Klaus, J., Zagler, W.L., Burger, D. (eds.) ICCHP 2004. LNCS, vol. 3118, pp. 522-529. Springer, Heidelberg (2004)

6. Bigham, J.P., Cavender, A.C., Brudvik, J.T., Wobbrock, J.O., Lander, R.E.: Webinsitu: A comparative analysis of blind and sighted browsing behavior. In: Proceedings of the 9th International ACM SIGACCESS Conference on Computers and Accessibility, Assets 2007, pp. 51-58. ACM, New York (2007)

7. Vigo, M., Harper, S.: Coping tactics employed by visually disabled users on the web. Int. J. Hum. -Comput. Stud. 71(11), 1013-1025 (2013)

8. Lazarus, R.S., Folkman, S.: Stress, appraisal, and coping. Springer Pub. Co., New York (1984) 
9. Takagi, H., Saito, S., Fukuda, K., Asakawa, C.: Analysis of navigability of web applications for improving blind usability. ACM Trans. Comput. -Hum. Interact. 14(3) (September 2007)

10. Leuthold, S., Bargas-Avila, J.A., Opwis, K.: Beyond web content accessibility guidelines: Design of enhanced text user interfaces for blind internet users. Int. J. Hum. -Comput. Stud. 66(4), 257-270 (2008)

11. Hillen, H., Evers, V.: Website navigation for blind users. John Wiley \& Sons, Ltd. (2007) ISBN-13: 978-0-470-01866-8

12. Grillo, F.D.N., Fortes, R.P.M.: Awmo: Accessible web modeler. In: WebMedia 2013 - 19th Brazilian Symposium on Multimedia and the Web - XVII Workshop on Tools and Applications (2013)

13. Fortes, R.P.M., Dias, A.L., Grillo, F.D.N., Masiero, P.C.: Creating a project towards universal access: is it possible? In: INTERACT 2013 Workshop on Rethinking Universal Accessibility: A Broader Approach Considering the Digital Gap, Cape Town, South Africa (2013)

14. Grillo, F.D.N., Fortes, R.P.M.: Accessible modeling on the web: a case study. In: Procedia Computer Science, Proceedings of the 5th International Conference on Software Development for Enhancing Accessibility and Fighting Info-exclusion, DSAI 2013 (2013) 